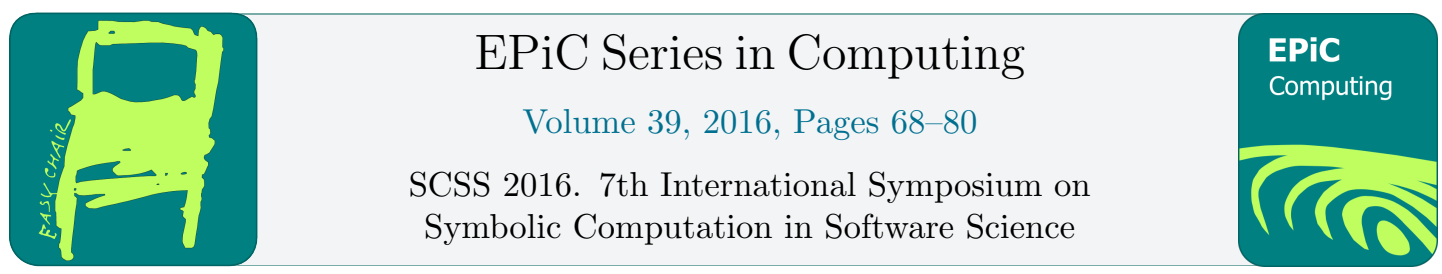

\title{
A Mathematica module for conformal geometric algebra and origami folding
}

\author{
Mitsuhiro Kondo ${ }^{1 *}$ Takuya Matsuo ${ }^{1}$, Yoshihiro Mizoguchi², and Hiroyuki \\ Ochiai $^{2}$ \\ 1 Graduate School of Mathematics, Kyushu University, Japan \\ 2 Institute of Mathematics for Industry, Kyushu University, Japan
}

\begin{abstract}
We implemented a Mathematica module for conformal geometric algebra (CGA) which includes functions to represent CGA elements and compute operations on the elements. In particular, we can draw a figure in 3D space corresponding to a CGA element. The proposed drawing function uses a Gröbner basis for simplifying the corresponding equations. This function can visualize any CGA element. One motivation of the present study is to realize a 3D origami system using our own CGA library. This $3 \mathrm{D}$ system is based on the 2D computational origami system E-Origami-System developed by Ida et al. and simple fold operations were formulated in 3D using CGA points and motions. We then prove geometric theorems concerning 3D origami properties using the proposed module.
\end{abstract}

\section{Introduction}

Complex numbers can be used to describe a point on a plane. The addition of complex numbers can be considered to be a translation of a point on a plane, while their multiplication can be considered to be a magnification, reduction, and rotation transformation of a point on a plane. Quaternions are a number system which is an extension of the complex numbers introduced by W.R. Hamilton in 1843. We can use the quaternion numbers to describe a point in a three-dimensional (3D) space. Calculation of a quaternion involves a rotation of a point in 3D space. Since it is easy to manipulate a computation using its algebraic properties, quaternions are used in applied mathematics, e.g., in 3D computer graphics and computer vision $[10,7,11]$. Conformal geometric algebra (CGA) is an extension of algebraic number systems which can involve space points, rotations, magnifications, reductions, and translations of higher-dimensional spaces $[1,12,9]$.

In the present paper, we investigate CGA to consider figures in $\mathbb{R}^{3}$ and their transformations. We herein consider a CGA as a 32-dimension linear space. Transformations of figures in $\mathbb{R}^{3}$ can be described using a $4 \times 4$ affine transformation matrix, and a point in a figure can be described using a vector in $\mathbb{R}^{3}$. Our motivation for using CGA is to describe both points and transformations in a single algebraic framework in order to manipulate the computation and to

*kondo.mitsuhiro@kyudai.jp 
easily verify its properties. One of our research goals is to find a useful algorithm for visualizing the basic geometric entities used in computer graphics through the concepts in CGA.

We implemented a Mathematica module which includes functions to represent CGA elements and to compute operations in CGA, such as the geometric product, the inner product, and the outer product. Furthermore, we can draw the figure in $\mathbb{R}^{3}$ which corresponds to a CGA element. Our drawing function uses a Gröbner basis for simplifying equations corresponding to figures. Moreover, our drawing function can also be used for multi-dimensional figures. We also implemented a function to check the equality of two figures which are represented by different CGA elements [8].

Some origami are made in 2D, as shown in Figure 1. Some of these origami, such as the crane origami, can be extended to 3D after all folding operations, but these are closed in 2D during folding. Since all fold operations are $\pi$ folding, 2D origami calculation is closed in 2D. However, there are some origami which cannot be obtained by $\pi$ folding alone. For example, in order to construct a piano, as shown in Figure 2, we need to use $\frac{\pi}{2}$ folding. We then consider an application of CGA to the 3D origami formalization and investigate its properties.

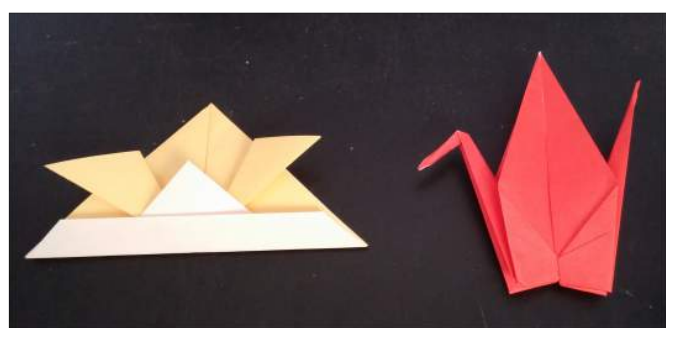

Figure 1: 2D Origami

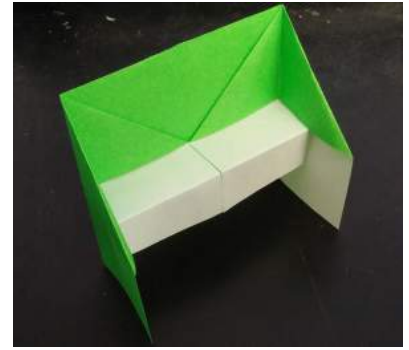

Figure 2: 3D Origami

Ida et al. formalized $2 \mathrm{D}$ origami development using the system Eos and verified a number of origami properties [3, 6, 5, 2]. In 2014, Ida introduced the concept of extending Eos to 3D origami [4]. Our goal is to realize a 3D origami system using our own CGA library. Based on the Eos 2D computational origami system, simple fold operations in $3 \mathrm{D}$ were formulated using CGA points and motions. We then proved a simple geometric theorem for 3D origami properties by calculating CGA equation formulas.

\section{Conformal geometric algebra}

Let $\mathcal{A}$ be the finite set $\{0,1,2,3, \infty\}$. We define sets of variables $W=\left\{w_{S} \mid S \subset \mathcal{A}\right\}$ and $E=\left\{e_{S} \mid S \subset \mathcal{A}\right\}$. A geometric algebra $G$ has six operators, Product $(*: G \times G \rightarrow G)$, Sum $(+: G \times G \rightarrow G)$, Minus $(-: G \rightarrow G)$, Outer product $(\wedge: G \times G \rightarrow G)$, Inner product $(\cdot: G \times G \rightarrow G)$, and Scalar product $(\cdot: \mathbb{R} \times G \rightarrow G)$. In order to describe an element of the geometric algebra $G$ as a polynomial in $\mathbb{R}[E]$ or $\mathbb{R}[W]$, we define some computing functions on $\mathbb{R}[E]$ and $\mathbb{R}[W]$. We assume that $w_{\{a\}}=e_{\{a\}}(a \in \mathcal{A})$, and

$$
e_{\{i\}} \cdot e_{\{j\}}= \begin{cases}1 & (i=j \wedge(1 \leq i, j \leq 3)) \\ 0 & (i \neq j \wedge(1 \leq i, j \leq 3)) \vee(i=j=0) \vee(i=j=\infty) \\ -1 & ((i=0 \wedge j=\infty) \vee(i=\infty \wedge j=0))\end{cases}
$$


In order to compute a product of two polynomials in $\mathbb{R}[E]$, we define

$$
\begin{aligned}
e_{S} * e_{\phi} & =e_{\phi} * e_{S}=e_{S}, \\
e_{\{0\}} * e_{S} & = \begin{cases}e_{\{0\} \cup S} & (0 \notin S) \\
0 & (0 \in S),\end{cases} \\
e_{\{a\}} * e_{S} & = \begin{cases}(-1)^{|\{s \in S \mid s<a\}|} e_{\{a\} \cup S} & (a \in\{1,2,3\} \wedge a \notin S) \\
(-1)^{|\{s \in S \mid s<a\}|} e_{S-\{a\}} & (a \in\{1,2,3\} \wedge a \in S),\end{cases} \\
e_{\{\infty\}} * e_{S} & = \begin{cases}(-1)^{|S|} e_{\{\infty\} \cup S} & (0 \notin S \wedge \infty \notin S) \\
0 & (0 \notin S \wedge \infty \in S) \\
-e_{\{0\}} *\left(e_{\{\infty\}} * e_{S-\{0\}}\right)-2 e_{S-\{0\}} & (0 \in S),\end{cases} \\
e_{T \cup\{a\}} * e_{S} & =e_{T} *\left(e_{\{a\}} * e_{S}\right)(\forall t \in T, t<a) .
\end{aligned}
$$

For computing a product of two polynomials in $\mathbb{R}[W]$, we use the following transformation and computations in $\mathbb{R}[E]$.

$$
w_{S}= \begin{cases}e_{S} & (0 \notin S \vee \infty \notin S) \\ e_{S}+(-1)^{|S|} e_{S-\{0, \infty\}} & (0 \in S \wedge \infty \in S) .\end{cases}
$$

We define the function grade $: \mathbb{R}[W] \rightarrow \mathbb{N}$ by $\operatorname{grade}\left(a w_{S}\right)=|S|(a \in \mathbb{R})$ and $\operatorname{grade}(f+g)=$ $\max ($ grade $(f)$, grade $(g))(f, g \in \mathbb{R}[W])$. For $k \in \mathbb{N}$, the function pickup $k: \mathbb{R}[W] \rightarrow \mathbb{R}[W]$ identifies terms which have grade $(f)=k(f \in \mathbb{R}[W])$. That is, pickup $2\left(w_{0}+w_{01}+w_{012}+w_{12}\right)=$ $w_{01}+w_{12}$. Note that $w_{\phi}, w_{\{0\}}, w_{\{0,1\}}$, and $w_{\{0,1,2\}}$ are denoted by $1, w_{0}, w_{01}$, and $w_{012}$, respectively. In order to compute an outer product $(\wedge)$ and an inner $\operatorname{product}(\cdot)$ in $\mathbb{R}[W]$, we define

$$
\begin{aligned}
w_{S} \wedge w_{T} & =\operatorname{pickup}_{\left|\operatorname{grade}\left(w_{S}\right)+\operatorname{grade}\left(w_{T}\right)\right|}\left(w_{S} * w_{T}\right), \text { and } \\
w_{S} \cdot w_{T} & =\operatorname{pickup}_{\left|\operatorname{grade}\left(w_{S}\right)-\operatorname{grade}\left(w_{T}\right)\right|}\left(w_{S} * w_{T}\right) .
\end{aligned}
$$

Note that $x * y=x \cdot y+x \wedge y$ for any elements $x, y \in \mathbb{R}[W]$. In Tables 1 and 2 , we list the operation tables of the product and the outer product, respectively.

Table 1: Product operation table

\begin{tabular}{c||c|c|c|c|c}
$*$ & $e_{0}$ & $e_{1}$ & $e_{2}$ & $e_{3}$ & $e_{\infty}$ \\
\hline \hline$e_{0}$ & 0 & $e_{01}$ & $e_{02}$ & $e_{03}$ & $e_{0 \infty}$ \\
\hline$e_{1}$ & $-e_{01}$ & 1 & $e_{12}$ & $e_{13}$ & $e_{1 \infty}$ \\
\hline$e_{2}$ & $-e_{02}$ & $-e_{12}$ & 1 & $e_{23}$ & $e_{2 \infty}$ \\
\hline$e_{3}$ & $-e_{03}$ & $-e_{13}$ & $-e_{23}$ & 1 & $e_{3 \infty}$ \\
\hline$e_{\infty}$ & $-2-e_{0 \infty}$ & $-e_{1 \infty}$ & $-e_{2 \infty}$ & $-e_{3 \infty}$ & 0 \\
\hline
\end{tabular}

\begin{tabular}{c||c|c|c|c|c}
$*$ & $w_{0}$ & $w_{1}$ & $w_{2}$ & $w_{3}$ & $w_{\infty}$ \\
\hline \hline$w_{0}$ & 0 & $w_{01}$ & $w_{02}$ & $w_{03}$ & $-1+w_{0 \infty}$ \\
\hline$w_{1}$ & $-w_{01}$ & 1 & $w_{12}$ & $w_{13}$ & $w_{1 \infty}$ \\
\hline$w_{2}$ & $-w_{02}$ & $-w_{12}$ & 1 & $w_{23}$ & $w_{2 \infty}$ \\
\hline$w_{3}$ & $-w_{03}$ & $-w_{13}$ & $-w_{23}$ & 1 & $w_{3 \infty}$ \\
\hline$w_{\infty}$ & $-1-w_{0 \infty}$ & $-w_{1 \infty}$ & $-w_{2 \infty}$ & $-w_{3 \infty}$ & 0 \\
\hline
\end{tabular}

A function exp on a CGA is defined by a formal power series $\exp (x)=\sum_{k=0}^{\infty} \frac{1}{k !} x^{k}$ where we abbreviate a product operator symbol $*$. We note that $\exp (x) \in \mathbb{R}[W]$ for any $x \in \mathbb{R}[W]$.

There are known relationships between CGA elements and figures in $\mathbb{R}^{3}$. A point $(x, y, z) \in$ $\mathbb{R}^{3}$ is represented by a CGA element $P_{(x, y, z)}=w_{0}+x w_{1}+y w_{2}+z w_{3}+\frac{1}{2}\left(x^{2}+y^{2}+z^{2}\right) w_{\infty}$. 
Table 2: OuterProduct operation table

\begin{tabular}{c||c|c|c|c|c}
$\wedge$ & $e_{0}$ & $e_{1}$ & $e_{2}$ & $e_{3}$ & $e_{\infty}$ \\
\hline \hline$e_{0}$ & 0 & $e_{01}$ & $e_{02}$ & $e_{03}$ & $1+e_{0 \infty}$ \\
\hline$e_{1}$ & $-e_{01}$ & 0 & $e_{12}$ & $e_{13}$ & $e_{1 \infty}$ \\
\hline$e_{2}$ & $-e_{02}$ & $-e_{12}$ & 0 & $e_{23}$ & $e_{2 \infty}$ \\
\hline$e_{3}$ & $-e_{03}$ & $-e_{13}$ & $-e_{23}$ & 0 & $e_{3 \infty}$ \\
\hline$e_{\infty}$ & $-1-e_{0 \infty}$ & $-e_{1 \infty}$ & $-e_{2 \infty}$ & $-e_{3 \infty}$ & 0 \\
\hline
\end{tabular}

\begin{tabular}{c||c|c|c|c|c}
$\wedge$ & $w_{0}$ & $w_{1}$ & $w_{2}$ & $w_{3}$ & $w_{\infty}$ \\
\hline \hline$w_{0}$ & 0 & $w_{01}$ & $w_{02}$ & $w_{03}$ & $w_{0 \infty}$ \\
\hline$w_{1}$ & $-w_{01}$ & 0 & $w_{12}$ & $w_{13}$ & $w_{1 \infty}$ \\
\hline$w_{2}$ & $-w_{02}$ & $-w_{12}$ & 0 & $w_{23}$ & $w_{2 \infty}$ \\
\hline$w_{3}$ & $-w_{03}$ & $-w_{13}$ & $-w_{23}$ & 0 & $w_{3 \infty}$ \\
\hline$w_{\infty}$ & $-w_{0 \infty}$ & $-w_{1 \infty}$ & $-w_{2 \infty}$ & $-w_{3 \infty}$ & 0 \\
\hline
\end{tabular}

A circle passing through three points $a, b$, and $c$ is $P_{a} \wedge P_{b} \wedge P_{c}$. A sphere passing through four points $a, b, c$, and $d$ is $P_{a} \wedge P_{b} \wedge P_{c} \wedge P_{d}$. A line passing through two points $a$ and $b$ is $P_{a} \wedge P_{b} \wedge w_{\infty}$. A plane passing through three points $a, b$, and $c$ is $P_{a} \wedge P_{b} \wedge P_{c} \wedge w_{\infty}$. A translator is defined by $\exp \left(-\frac{d}{2} w_{\infty}\right) P \exp \left(\frac{d}{2} w_{\infty}\right)$ where $d=\alpha w_{1}+\beta w_{2}+\gamma w_{3},(\alpha, \beta, \gamma \in \mathbb{R})$. A rotor is defined by $\exp \left(-\frac{\theta}{2} B\right) P \exp \left(\frac{\theta}{2} B\right)$, where $\theta \in \mathbb{R}$ is an angle of rotation, $B=b_{1} \wedge b_{2}$, and $b_{i}=\alpha_{i} w_{1}+\beta_{i} w_{2}+\gamma_{i} w_{3},\left(\alpha_{i}, \beta_{i}, \gamma_{i} \in \mathbb{R}, i=1,2\right)$. A dilator is $\exp \left(-\frac{\lambda}{2} w_{0} \wedge w_{\infty}\right) P \exp \left(\frac{\lambda}{2} w_{0} \wedge w_{\infty}\right)$, where $\lambda \in \mathbb{R}$ is a scaling factor.

Example 1. In the following, we show some simple computations of elements in $W$ :

$$
\begin{aligned}
w_{01}=w_{0} \wedge w_{1} & =-w_{1} \wedge w_{0}, \\
w_{\infty} * w_{0} & =-1-w_{0 \infty}, \\
w_{0 \infty} * w_{123} & =-w_{023 \infty}, \\
w_{12} * w_{3 \infty} & =w_{123 \infty}, \\
w_{3 \infty} * w_{3 \infty} & =\left(w_{3 \infty}\right)^{2}=0, \\
w_{0 \infty} * w_{0 \infty} & =\left(w_{0 \infty}\right)^{2}=0, \\
w_{12} * w_{12} & =\left(w_{12}\right)^{2}=-1, \\
w_{12} & =e_{12}, \text { and } \\
w_{01 \infty} & =e_{01 \infty}-e_{1} .
\end{aligned}
$$

The following examples are computations of the exp function. Since $\left(w_{3 \infty}\right)^{2}=0,\left(w_{12}\right)^{2}=$ -1 and $\left(w_{0 \infty}\right)^{2}=1$, we have

$$
\begin{aligned}
\exp \left(\frac{w_{3}}{2} w_{\infty}\right) & =1+\frac{1}{2} w_{3 \infty}+\frac{1}{2 !}\left(\frac{1}{2} w_{3 \infty}\right)^{2}+\cdots \\
& =1+\frac{1}{2} w_{3 \infty}, \\
\exp \left(\frac{\theta}{2} w_{12}\right) & =1+\frac{\theta}{2} w_{12}+\frac{1}{2 !}\left(\frac{\theta}{2} w_{12}\right)^{2}+\frac{1}{3 !}\left(\frac{\theta}{2} w_{12}\right)^{3}+\cdots \\
& =\left\{1-\frac{1}{2 !}\left(\frac{\theta}{2}\right)^{2}+\cdots\right\}+\left\{\frac{\theta}{2}-\frac{1}{3 !}\left(\frac{\theta}{2}\right)^{3}+\cdots\right\} w_{12} \\
& =\cos \frac{\theta}{2}+w_{12} \sin \frac{\theta}{2}, \text { and } \\
\exp \left(\frac{\lambda}{2}\left(1+w_{\infty} w_{0}\right)\right) & =\exp \left(-\frac{\lambda}{2} w_{0 \infty}\right) \\
& =1+\frac{\lambda}{2} w_{0 \infty}+\frac{1}{2 !}\left(\frac{\lambda}{2} w_{0 \infty}\right)^{2}+\frac{1}{3 !}\left(\frac{\lambda}{2} w_{0 \infty}\right)^{3}+\cdots
\end{aligned}
$$




$$
\begin{aligned}
& =\left\{1+\frac{1}{2 !}\left(\frac{\lambda}{2}\right)^{2}+\cdots\right\}+\left\{\frac{\lambda}{2}+\frac{1}{3 !}\left(\frac{\lambda}{2}\right)^{3}+\cdots\right\} w_{0 \infty} \\
& =\cosh \frac{\lambda}{2}-w_{0 \infty} \sinh \frac{\lambda}{2} .
\end{aligned}
$$

\section{Mathematica module for CGA}

The proposed Mathematica module has various functions. For example, CGAProduct, OuterProduct and InnerProduct are functions for computing products $(*, \wedge$ and $\cdot)$ of two CGA elements. Moreover, Pnt, Cir, Trs, etc. are functions for creating a CGA element which represents a point, a circle, a translator, etc. The figure correspoinding to a CGA element $X$ is a subset $\operatorname{Fig}(X):=\left\{(x, y, z) \in \mathbb{R}^{3} \mid X \wedge P_{(x, y, z)}=0\right\}$ of $\mathbb{R}^{3}$. We implemented a function CGAOutput $3 D$ for drawing the corresponding figure in $\mathbb{R}^{3}$ for a given CGA element. CGAEquationCheck is a function for checking the equality of figures represented by two CGA elements. Let $X=\sum_{S \subset \mathcal{A}} a_{S} w_{S} \quad\left(a_{S} \in \mathbb{R}\right)$. Then we have $X \wedge P_{(x, y, z)}=\sum_{S \subset \mathcal{A}} p_{S} w_{S}$, where $p_{S}$ are polynomials in $\mathbb{R}[x, y, z]$ and $\operatorname{Fig}(X)=\left\{(x, y, z) \mid p_{S}=0(S \subset \mathcal{A})\right\}$. We list complete equations for $F i g(X)$ in Appendix. We compute a Gröbner basis of those equations. We note that there remains at most one degree 2 equation in the Gröbner basis for this case. So we can detect the dimension of a figure by the number of elements in the Gröbner basis. That is the dimension is 3 minus the number of elements. If the number is three, then the figure is a finite set of points. If the number is two, then we can draw the figure using ParametricPlot $3 D$ function with one variable. If the number is one, then we use ContourPlot $3 D$ function with two variables. Our CGAOutput $3 D$ function automatically choose an appropriate draw function in Mathematica.

We show two examples. First, we consider the intersection of a sphere and a plane. Let $S_{1}$ be the sphere passing through four points $(3,0,0),(0,3,1),(0,2,3)$, and $(-3,3,2), H_{1}$ the plane which passes through three points $(3,0,1),(0,3,1)$, and $(0,2,1)$. That is, $S_{1}=P_{(3,0,0)}$ $\wedge P_{(0,3,1)} \wedge P_{(0,2,3)} \wedge P_{(-3,3,2)}=3 w_{012 \infty}-33 w_{013 \infty}+37 w_{023 \infty}+192 w_{123 \infty}-18 w_{0123}$ and $H_{1}$ $=P_{(3,0,1)} \wedge P_{(0,3,1)} \wedge P_{(0,2,1)} \wedge w_{\infty}=3 w_{012 \infty}+3 w_{123 \infty}$. The intersection of $S_{1}$ and $H_{1}$ is a CGA element $S_{1} \cdot H_{1}^{*}=-99 w_{01 \infty}+111 w_{02 \infty}+567 w_{12 \infty}+99 w_{13 \infty}-111 w_{23 \infty}+54 w_{012}+54 w_{123}$, where $H_{1}^{*}$ is the dual of $H_{1}$ i.e. $H_{1}^{*}=H_{1} *\left(-w_{0123 \infty}\right)$ (cf. [12]). Figure 3 represents the intersection of plane $H_{1}$ and sphere $S_{1}$.

$$
\begin{aligned}
\text { Fig }\left(S_{1} \cdot H_{1}^{*}\right) & =\left\{(x, y, z) \in \mathbb{R}^{3} \mid-1+z=0,-180+37 x+9 x^{2}+33 y+9 y^{2}=0\right\} \\
& =\left\{\left(x,-\frac{11}{6} \pm \frac{1}{6} \sqrt{841-148 x-36 x^{2}}, 1\right) \in \mathbb{R}^{3} \mid x \in \mathbb{R} \wedge 841-148 x-36 x^{2}>0\right\} .
\end{aligned}
$$

Since the number of elements in the Gröbner basis is two, an element in the set is expressed with one variable. In this example, we can draw a circle using the Mathematica function ParametricPlot3D.

Next, we consider the intersection of a circle and a plane. Let $C_{1}$ be the circle which passes through three points $(3,0,2),(0,3,1)$, and $(0,3,0)$, that is $C_{1}=P_{(3,0,2)} \wedge P_{(0,3,1)} \wedge P_{(0,3,0)}$ $=\frac{3}{2} w_{01 \infty}-\frac{3}{2} w_{02 \infty}-w_{03 \infty}-\frac{9}{2} w_{12 \infty}+\frac{27}{2} w_{13 \infty}-\frac{33}{2} w_{23 \infty}+3 w_{013}-3 w_{023}-9 w_{123}$. The intersection of plane $H_{1}$ and circle $C_{1}$ is a CGA element $C_{1} \cdot H_{1}^{*}=-3 w_{0 \infty}+45 w_{1 \infty}-54 w_{2 \infty}-$ $3 w_{3 \infty}-9 w_{01}+9 w_{02}+27 w_{12}+9 w_{13}-9 w_{23}$.

$$
\begin{aligned}
F i g\left(C_{1} \cdot H_{1}^{*}\right) & =\left\{(x, y, z) \in \mathbb{R}^{3} \mid-1+z=0,-3-8 y+3 y^{2}=0,-3+x+y=0\right\} \\
& =\left\{\left(\frac{5+\sqrt{7}}{3}, \frac{4-\sqrt{7}}{3}, 1\right),\left(\frac{5-\sqrt{7}}{3}, \frac{4+\sqrt{7}}{3}, 1\right)\right\} .
\end{aligned}
$$


Since the number of elements in the Gröbner basis is three, we can completely solve the equations. Therefore, we draw these points using the Mathematica function ListPointPlot3D. Figure 4 represents the intersection of plane $H_{1}$ and circle $C_{1}$.

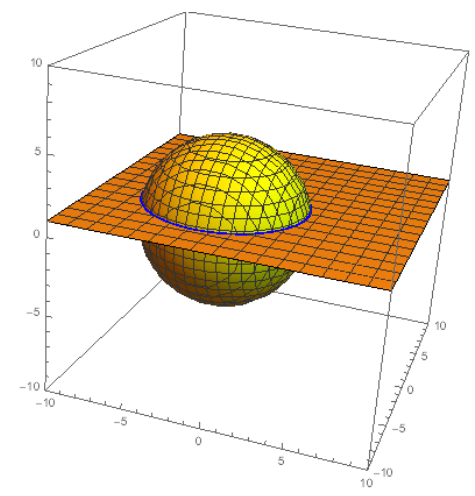

Figure 3: Sphere and Plane

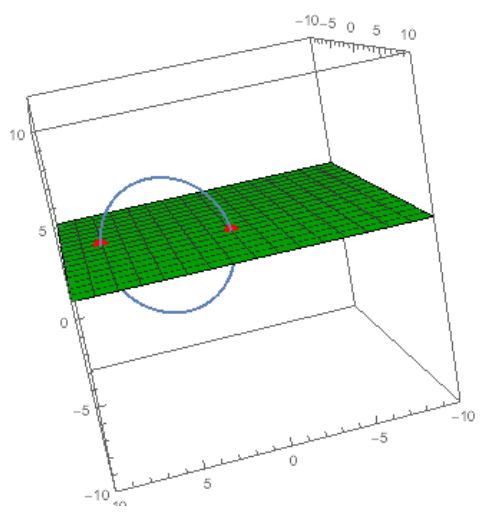

Figure 4: Plane and Circle

We also implemented a function to check whether the figures of two CGA elements are equal. Let $X_{1}=w_{0 \infty}-6 w_{1 \infty}-5 w_{2 \infty}-4 w_{3 \infty}+w_{01 \infty}+w_{02 \infty}+w_{03 \infty}+6 w_{12 \infty}+5 w_{13 \infty}+6 w_{23 \infty}$ $+w_{012 \infty}+w_{013 \infty}+w_{023 \infty}-5 w_{123 \infty}+w_{0123 \infty}+w_{01}+w_{02}+w_{03}-w_{12}-2 w_{13}-w_{23}+w_{012}$ $+w_{013}+w_{023}+2 w_{123}+w_{0123}$ and $X_{2}=w_{0}+w_{1}+2 w_{2}+3 w_{3}+7 w_{\infty}$. The appearances of $X_{1}$ and $X_{2}$ are different, but the figures of $X_{1}$ and $X_{2}$ are same and it is $\{(1,2,3)\}$. Our implemented function CGAEquationCheck can check the equality of figures for any two CGA elements using Gröbner bases and the PolynomialMod function in Mathematica.

\section{Two-dimensional origami folding}

We follow the formulations introduced by Ida et al. [6, 13, 5, 2]. First, we use a data structure called origami graph $O=(\Pi, \sim, \succ)$, where $\Pi$ is a set of faces, $\sim$ is an adjacency relation of faces, and $\succ$ is a superposition relation (cf. [13, 5]).

Our Mathematica module has various functions. Ori is the function that folds origami and automatically updates the origami graph $O$. Arguments of $\operatorname{Ori}[O, m, F, \theta]$ are a current origami graph $O=(\Pi, \sim, \succ)$, a fold line $m$, a faces set $F$ for folding and an angle of rotation $\theta \in\{\pi,-\pi, 0\}$, where $F$ may be a part of faces for folding and $\theta$ indicates a valley fold or a mountain fold. We use $\theta=0$ for an operation which does not fold but just divide faces using a given fold line and a face set. To determine a folding line from given points and lines, we follow the Huzita-Hattori axioms and we implemented Mathematica functions Ori1 to Ori\%. We recall the Huzita-Hatori axioms using our functions as follows[2]:

Axiom 1: Given two points $p_{1}$ and $p_{2}$, there is a unique fold line (Ori1 $\left.\left[p_{1}, p_{2}\right]\right)$ that passes through both points (Figure 5).

Axiom 2: Given two points $p_{1}$ and $p_{2}$, there is a unique fold line (Ori2 $\left.\left[p_{1}, p_{2}\right]\right)$ that places $p_{1}$ onto $p_{2}$ (Figure 6).

Axiom 3: Given two lines $L_{1}$ and $L_{2}$, there are at most two fold lines (Ori3 $\left[L_{1}, L_{2}\right.$, flag]) that place $L_{1}$ onto $L_{2}$. There are at most two choices of folding line. We use "flag" to indicate a folding line(Figure 7). 
Axiom 4: Given a point $p_{1}$ and a line $L_{1}$, there is a unique fold line (Ori4 $\left[p_{1}, L_{1}\right]$ ) that passes through point $p_{1}$ and is perpendicular to $L_{1}$ (Figure 8).

Axiom 5: Given two points $p_{1}$ and $p_{2}$ and a line $L_{1}$, there are at most two fold lines (Ori5 $\left[p_{1}, p_{2}, L_{1}\right.$, flag $]$ ) that place $p_{2}$ onto $L_{1}$ and pass through $p_{1}$. There are at most two choices of folding line. We use "flag" to indicate a folding line (Figure 9).

Axiom 6: Given two points $p_{1}$ and $p_{2}$ and two lines $L_{1}$ and $L_{2}$, there are at most three fold lines (Ori6 $\left[p_{1}, L_{1}, p_{2}, L_{2}\right.$, flag]) that place $p_{1}$ onto $L_{1}$ and $p_{2}$ onto $L_{2}$. There are at most three choices of folding line. We use "flag" to indicate a folding line (Figure 10).

Axiom 7: Given one point $p$ and two lines $L_{1}$ and $L_{2}$, there is a unique fold line (Ori7 $\left[p_{1}, L_{1}, L_{2}\right]$ ) that places $p$ onto $L_{2}$ and is perpendicular to $L_{1}$ (Figure 11 ).

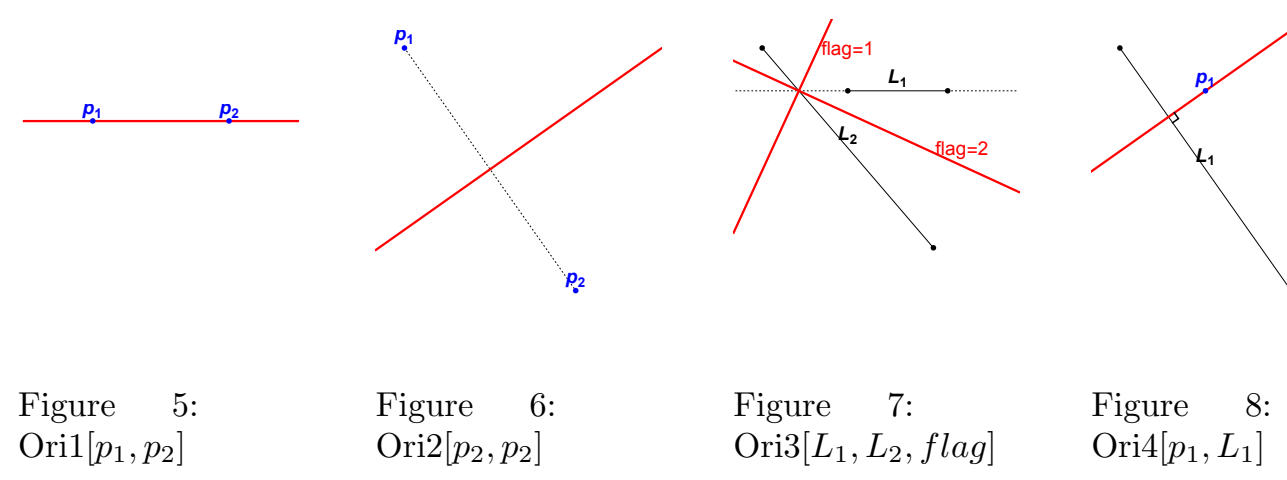

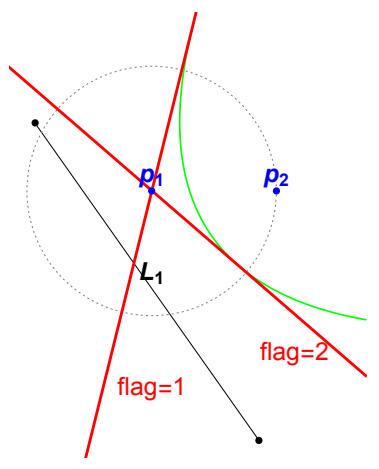

Figure $\quad 9$ :

Ori5 $\left[p_{1}, p_{2}, L_{1}\right.$, flag $]$

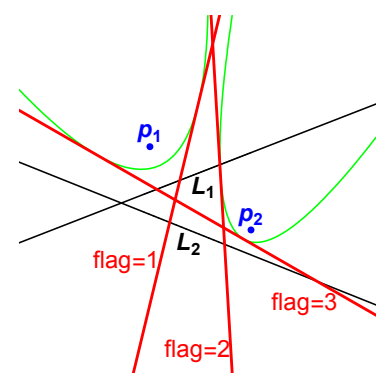

Figure 10 :

Ori6 $\left[p_{1}, L_{1}, p_{2}, L_{2}\right.$, flag $]$

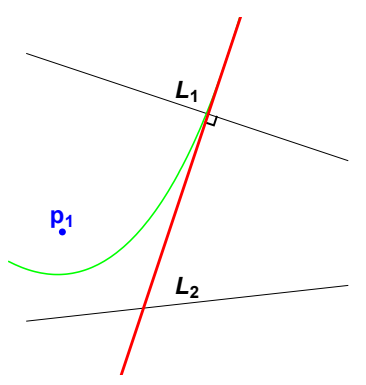

Figure 11 :

$\operatorname{Ori7}\left[p_{1}, L_{1}, L_{2}\right]$

Example 2. We can make any origami by successive applications of origami functions. The following is a sequence of origami functions for making Kabuto. Intermediate results are displayed in Figures 12 to 15. 
We first define coordinates of vertices $\left(P_{1}, P_{2}, P_{3}, P_{4}\right)$ of Origami.

$$
\begin{aligned}
& P_{1}=\{0,0\} ; P_{2}=\{0,10\} ; P_{3}=\{10,10\} ; P_{4}=\{10,0\} ; \\
O_{0}= & \text { FirstO; } \\
O_{1}= & \operatorname{Ori}\left[O_{0}, \operatorname{Ori2}\left[P_{3}, P_{1}\right],\{1\}, \pi\right] ; \\
O_{2}= & \operatorname{Ori}\left[O_{1}, \operatorname{Ori} 2\left[P_{4}, P_{3}\right],\{3\}, \pi\right] ; \\
O_{3}= & \operatorname{Ori}\left[O_{2}, \operatorname{Ori2} 2\left[P_{1}, P_{4}\right],\{5\},-\pi\right] ;
\end{aligned}
$$

In this example, we explicitly define points $P_{5}, P_{6}$, and $P_{7}$, but these points are automatically defined after folding operations as the coordinate of a vertex of some folded face.

$$
\begin{aligned}
& P_{5}=\frac{P_{1}+P_{3}}{2} ; P_{6}=\frac{P_{1}+P_{2}}{2} ; P_{7}=\frac{P_{1}+P_{4}}{2} ; \\
O_{4}= & \left.\operatorname{Ori}\left[O_{3}, \text { Ori2 } 2 P_{5}, P_{1}\right],\{4,10\}, \pi\right] ; \\
O_{5}= & \left.\operatorname{Ori}\left[O_{4}, \text { Ori2 } 2 P_{6}, P_{5}\right],\{13\}, 0\right] ; \\
O_{6}= & \operatorname{Ori}\left[O_{5}, \operatorname{Ori} 2\left[P_{7}, P_{5}\right],\{29\}, 0\right] ; \\
& P_{8}=\frac{P_{1}+P_{5}}{2} ; P_{9}=\frac{P_{5}+P_{6}}{2} ; P_{10}=\frac{P_{5}+P_{7}}{2} ; \\
O_{7}= & \text { Ori }\left[O_{6}, \text { Ori3 }\left[\mathrm{p} 2 \operatorname{line}\left[P_{8}, P_{5}\right], \mathrm{p} 2 \operatorname{line}\left[P_{9}, P_{8}\right], 2\right],\{27\}, \pi\right] ; \\
O_{8}= & \text { Ori }\left[O_{7}, \text { Ori3 }\left[\mathrm{p} 2 \operatorname{line}\left[P_{8}, P_{5}\right], \text { p2line }\left[P_{10}, P_{8}\right], 2\right],\{59\}, \pi\right] ;
\end{aligned}
$$

The $P_{11}$ is a user defined point. In this example, we define $P_{11}$ as a midpoint of $P_{8}$ and $P_{5}$.

$$
\begin{aligned}
& P_{11}=\frac{P_{8}+P_{5}}{2} \\
& \left.O_{9}=\operatorname{Ori}\left[O_{8} \text {, Ori2 }\left[P_{11}, P_{1}\right],\{15\}, \pi\right] ; \quad \cdots \text { (Figure } 14\right) . \\
& O_{10}=\operatorname{Ori}\left[O_{9}, \operatorname{Ori1}\left[P_{7}, P_{6}\right],\{31\}, \pi\right] \\
& O_{11}=\operatorname{Ori}\left[O_{10}, \text { Ori2 }\left[P_{7}, P_{6}\right],\{11\},-\pi\right] ; \quad \cdots(\text { Figure } 15) \text {. }
\end{aligned}
$$
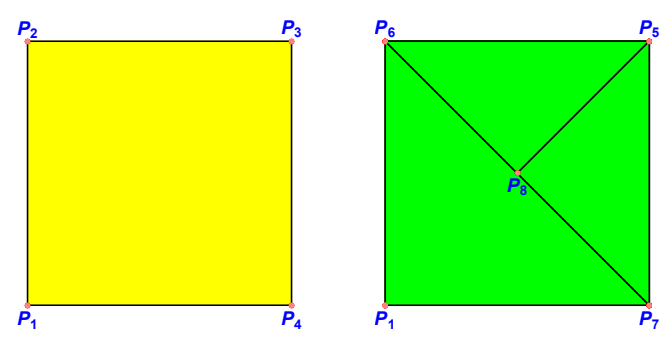

Figure 12: GOutput2D $\left(O_{0}\right)$

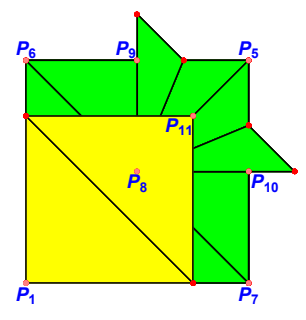

Figure 14: GOutput2D $\left(\mathrm{O}_{9}\right)$

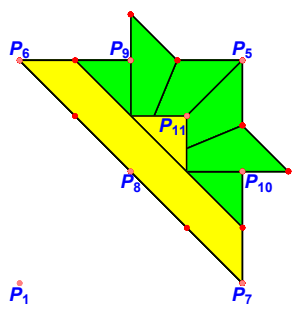

Figure 15 : GOutput2D $\left(O_{11}\right)$

GOutput2D is the function to draw an origami graph $O$, as viewed from the top and in a tab layer display in a 2D space (Figure 16). GOutput3D is the function to draw an origami graph 
$O$ by layer display in a $3 \mathrm{D}$ space (Figure 17). OriBack is the function to open folded origami, that is, the converse operation of Ori. This function can create a development view.

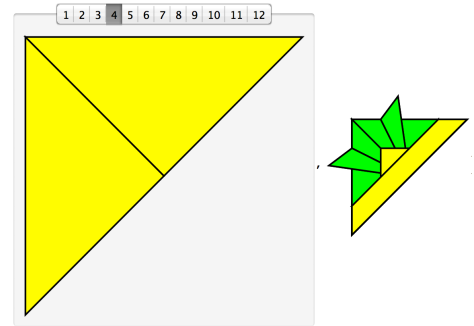

Figure 16: GOutput2D

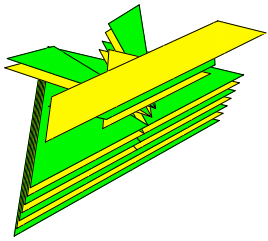

Figure 17: GOutput3D

\section{Three-dimensional origami folding}

When we transform a point $x$ to a point $y$, there are various types of representations that can be used to express a transformation. We often use a vector-matrix representation. We express operation $M$ as a $3 \times 3$ matrix and point $x, y$ as a $3 \times 1$ vector. A transform is represented as an equation $M x=y$. This representation requires the definition of two data structures (matrix and vector). We should calculate the act operators and product operator between two data types. Therefore, this is sometimes difficult to verify formally. However, CGA representation requires defining only one data structure (a set of CGA elements). In CGA representation, we can express both operation $R$ and point $x, y$ as a CGA element. Moreover, the transformation of point $x$ to point $y$ is represented as an algebraic equation $R * x * R^{-1}=y\left(R * R^{-1}=R^{-1} * R=1\right)$. Therefore, all calculations can be achieved simply by symbolic computation of the product operation $*$, making the operation easy to formalize and verify. This is the reason why we use our CGA representation.

We express the method by which to calculate a CGA operation $R$ from given fold operation as fold line $m$ and angle $\theta \in \mathbb{R}$. We consider fold line $m$, which is a directed line, as a pair of vectors $\left(v_{1}, v_{2}\right)$, and the direction is given by the vector $v=\frac{v_{2}-v_{1}}{\left\|v_{2}-v_{1}\right\|}$. A fold operation can be represented as the rotor on fold line $m$. As such, we define the rotor around the origin Rot and translator $T$ as follows:

$$
\begin{aligned}
\text { Rot }= & \cos \frac{\theta}{2}-B \sin \frac{\theta}{2} \\
& \left(\text { bivector } B=v *\left(-w_{123}\right) \text { which is the plane of the rotation }\right), \\
T= & 1-\frac{v_{1}}{2} w_{\infty} .
\end{aligned}
$$

Since $R o t$ is a rotor around the origin, we should rewrite the equation as a rotor around $v_{1}$. This can be achieved by using a translation defined by $T$ and $T^{-1}$. We define $R=T * R o t * T^{-1}$, where $R$ corresponds to the fold operation. This is the rotor of the left-hand direction on fold line $m$.

Example 3. We present an example of the calculation of CGA operation $R$. Let the fold operation be a $\frac{\pi}{3}$ folding on fold line $m=\left(v_{1}, v_{2}\right),\left(v_{1}=6 w_{1}, v_{2}=4 w_{1}+10 w_{2}\right)$ (Figure 18). 

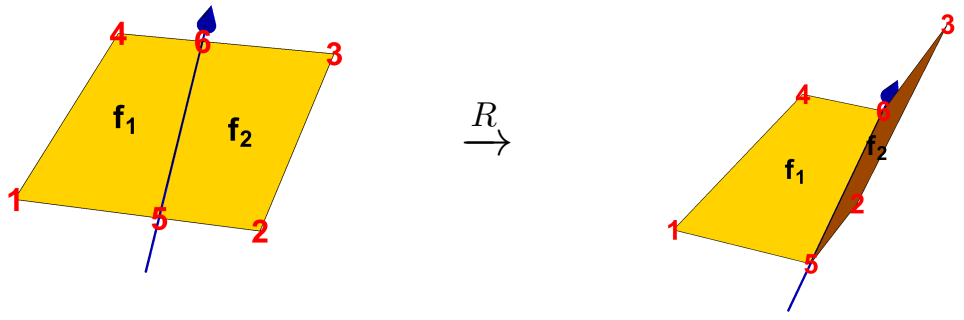

Figure 18: $\frac{\pi}{3}$ folding

$$
\begin{aligned}
v & =\frac{4 w_{1}+10 w_{2}-6 w_{1}}{\left\|4 w_{1}+10 w_{2}-6 w_{1}\right\|}=-\frac{w_{1}}{\sqrt{26}}+\frac{5 w_{2}}{\sqrt{26}} \\
B & =v *\left(-w_{123}\right)=\frac{5 w_{13}}{\sqrt{26}}+\frac{w_{23}}{\sqrt{26}} \\
R o t & =\cos \frac{\pi}{6}-B \sin \frac{\pi}{6}=\frac{\sqrt{3}}{2}-\frac{5 w_{13}}{2 \sqrt{26}}-\frac{w_{23}}{2 \sqrt{26}} \\
T & =1-3 w_{1 \infty}, T^{-1}=1+3 w_{1 \infty} \\
R & =T * \operatorname{Rot} * T^{-1}=\frac{\sqrt{3}}{2}-\frac{5 w_{13}}{2 \sqrt{26}}-\frac{w_{23}}{2 \sqrt{26}}+\frac{15 w_{3 \infty}}{\sqrt{26}}
\end{aligned}
$$

\section{Simple proof using CGA equations}

Various figures can be obtained by folding an origami. We can prove their geometric properties by calculating CGA equations. For example, when we fold a quadratic prism from rectangular origami (Figure 19), we show that points $P_{1}$ and $P_{2}$ are in the same position.

Let $P_{1}, P_{2}, P_{3}$, and $P_{4}$, which are the vertices of the rectangular origami, be the CGA points $P_{1}=P_{(0,0,0)}=w_{0}, P_{2}=P_{(A, 0,0)}=w_{0}+A w_{1}+\frac{A^{2}}{2} w_{\infty}, P_{3}=P_{(A, B, 0)}=w_{0}+A w_{1}+B w_{2}+$ $\frac{A^{2}+B^{2}}{2} w_{\infty}$ and $P_{4}=P_{(0, B, 0)}=w_{0}+B w_{2}+\frac{B^{2}}{2} w_{\infty}(A>0 \wedge B>0)$. We can express fold operations $R_{1}, R_{2}$, and $R_{3}$ in CGA and the CGA operation $R$ as a combination of $R_{1}, R_{2}$, and $R_{3}$, as follows: $R_{1}=\frac{1}{\sqrt{2}}+\frac{w_{13}}{\sqrt{2}}-\frac{A}{4 \sqrt{2}} w_{3 \infty}, R_{2}=\frac{1}{\sqrt{2}}+\frac{w_{13}}{\sqrt{2}}-\frac{A}{2 \sqrt{2}} w_{3 \infty}, R_{3}=\frac{1}{\sqrt{2}}+\frac{w_{13}}{\sqrt{2}}-\frac{3 A}{4 \sqrt{2}} w_{3 \infty}$, $R=R_{3} * R_{2} * R_{1}=\frac{1}{\sqrt{2}}+\frac{w_{13}}{\sqrt{2}}+\frac{A}{2 \sqrt{2}} w_{1, \infty}-\frac{A}{2 \sqrt{2}} w_{3 \infty}$.

We can calculate the CGA equation using the function in our Mathematica module

$$
R * P_{1} * R^{-1}=w_{0}+A w_{1}+\frac{A^{2}}{2} w_{\infty}=P_{2} .
$$

This equation confirms that points $P_{1}$ and $P_{2}$ are located at the same position. 


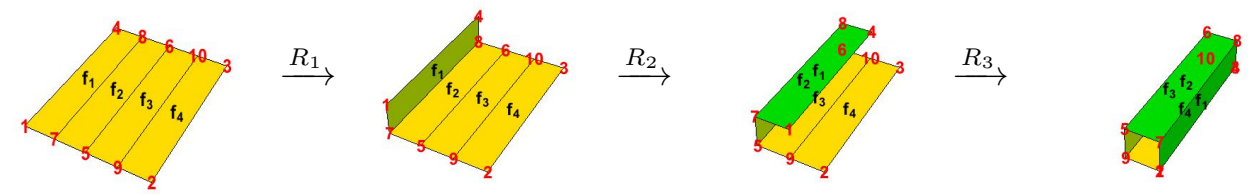

Figure 19: Folding the quadratic prism

\section{Conclusion}

We implemented functions that realize CGA operations using symbolic computations in Mathematica ${ }^{1}$. Our module includes functions for the geometric product, the inner product, and the outer product of a CGA which is an extension of $\mathbb{R}^{3}$ with $e_{0}$ and $e_{\infty}$. We also implemented a drawing function for figures in $\mathbb{R}^{3}$ which correspond to CGA elements. Furthermore, we implemented a function to check whether two figures of CGA elements are equal. Next, we considered an application of our CGA module to 3D origami folding. Following the formulation of $2 \mathrm{D}$ origami folding introduced by Ida et al., we extended the folding function. Using our module, we can study 3D origami folding, in particular the properties of a sequence of folding procedures. Finally, we presented a simple proof of the 3D origami property using a symbolic computation of the CGA equations. Future research include a precise implementation of 3D origami folding functions. We intend to implement a function for judging the collision of faces in $3 \mathrm{D}$ origami folding. Hopefully, we will be able to introduce various useful 3D origami motions described by CGA operations.

\section{Acknowledgement}

This work was partially supported by the Core Research for Evolutional Science and Technology (CREST) Program titled "Mathematics for Expressive Image Synthesis" of the Japan Science and Technology Agency (JST).

\section{References}

[1] P. Colapinto. Versor spatial computing with conformal geometric algebra. Master's thesis, University of California Santa Barbara, 2011.

[2] F. Ghourabi, A. Kasem, and C. Kaliszyk. Algebraic analysis of huzita's origami operations and their extensions. In Automated Deduction in Geometry, volume 7993 of Lecture Notes in Computer Science, pages 143-160. Springer, 2013.

[3] T. Ida. Eos project. http://www.i-eos.org:8080/ieos.

[4] T. Ida. Huzita's basic origami fold in geometric algebra. In 16th International Symposium on Symbolic and Numeric Algorithms for Scientific Computing (SYNASC 2014), pages 11-13. IEEE computer society, 2014.

[5] T. Ida and H. Takahashi. Origami fold as algebraic rewriting. Journal of Symbolic Computation, 45(4):393-413, 2010.

\footnotetext{
${ }^{1}$ cf. https://github.com/KyushuUniversityMathematics/MathematicaCGA
} 
[6] T. Ida, H. Takahashi, M. Marin, A. Kasem, and F. Ghourabi. Computational origami system eos. In Proc. 4th International Conference of Origami Science, Mathematics and Education (4OSME), pages 285-293, 2009.

[7] L. Kavan, S. Collins, J. Zara, and C. O'Sullivan. Geometric skinning with approximate dual quaternion blending. ACM Transaction on Graphics, 27(4):105:1-105:23, 2008.

[8] M. Kondo and T. Matsuo. A mathematica module for conformal geometric algebra. In Symposium on Mathematical Progress in Expressive Image Synthesis (MEIS2014), volume 58 of MI Lecture Note Series, pages 132 - 136. Kyushu University, 2014.

[9] A. Macdonald. A survey of geometric algebra and geometric calculus, 2014.

[10] G. Matsuda, S. Kaji, and H. Ochiai. Anti-commutative dual complex numbers and 2D rigid transofmation. In Symposium on Mathematical Progress in Expressive Image Synthesis (MEIS2013), MI Lecture Note Series, pages 128 - 133. Kyushu University, 2013.

[11] G. Papagiannakis. Geometric algebra rotors for skinned character animation blending. In SIGGRAPH Asia 2013 Technical Briefs, pages 11:1-11:6. ACM, 2013.

[12] C. Perwass. Geometric Algebra with Applications in Engineering, volume 4 of Geometry and Computing. Springer, 2009.

[13] H. Takahashi. A Study on algorithms for computational origami(Japanese). PhD thesis, University of Tsukuba, 2009. 


\section{Appendix}

Let $X=\sum_{S \subset \mathcal{A}} a_{S} w_{S},\left(a_{S} \in \mathbb{R}\right)$. Equations for $X \wedge P_{(x, y, z)}=0$ are listed as follows:

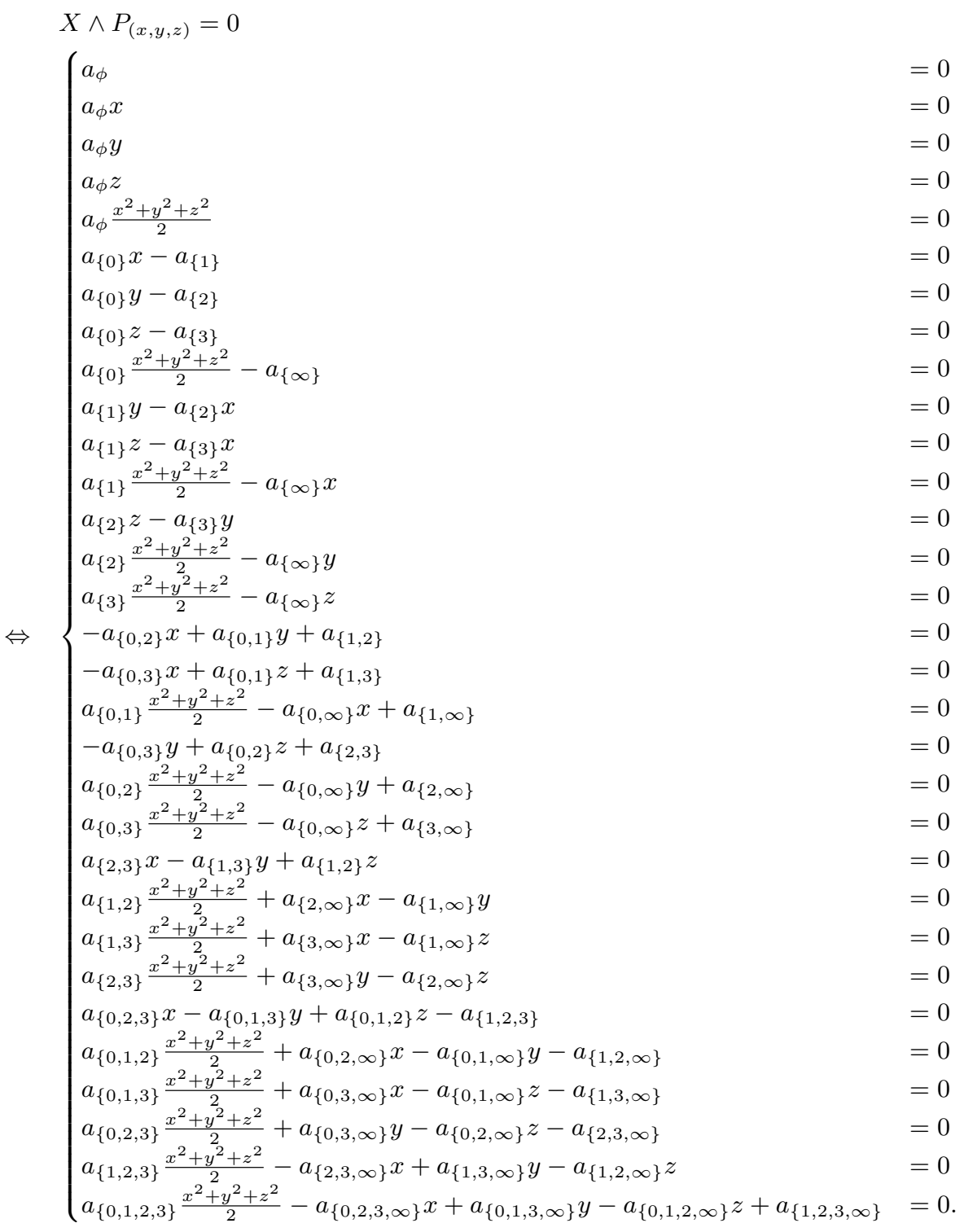

